\title{
Automatic Cell Segmentation by Adaptive Thresholding (ACSAT) for Large-Scale Calcium Imaging Datasets
}

\author{
Simon P. Shen, ${ }^{1}{ }^{*}$ Hua-an Tseng, ${ }^{2 *}$ Kyle R. Hansen, ${ }^{2}$ Ruofan $\mathrm{Wu}^{3}{ }^{3}$ CHoward J. Gritton, ${ }^{2}$ Jennie $\mathrm{Si}^{3}$ and \\ Xue $\operatorname{Han}^{2}$
}

https://doi.org/10.1523/ENEURO.0056-18.2018

${ }^{1}$ Department of Physics, Harvard University, Cambridge, MA 02138, ${ }^{2}$ Biomedical Engineering Department, Boston University, Boston, MA 02215, and ${ }^{3}$ School of Electrical, Computer and Energy Engineering, Arizona State University, Tempe, AZ 85287

\begin{abstract}
Advances in calcium imaging have made it possible to record from an increasingly larger number of neurons simultaneously. Neuroscientists can now routinely image hundreds to thousands of individual neurons. An emerging technical challenge that parallels the advancement in imaging a large number of individual neurons is the processing of correspondingly large datasets. One important step is the identification of individual neurons. Traditional methods rely mainly on manual or semimanual inspection, which cannot be scaled for processing large datasets. To address this challenge, we focused on developing an automated segmentation method, which we refer to as automated cell segmentation by adaptive thresholding (ACSAT). ACSAT works with a time-collapsed image and includes an iterative procedure that automatically calculates global and local threshold values during successive iterations based on the distribution of image pixel intensities. Thus, the algorithm is capable of handling variations in morphological details and in fluorescence intensities in different calcium imaging datasets. In this paper, we demonstrate the utility of ACSAT by testing it on 500 simulated datasets, two wide-field hippocampus datasets, a wide-field striatum dataset, a wide-field cell culture dataset, and a two-photon hippocampus dataset. For the simulated datasets with truth, ACSAT achieved $>80 \%$ recall and precision when the signal-to-noise ratio was no less than $\sim 24 \mathrm{~dB}$.
\end{abstract}

Key words: GCaMP6; genetically encoded calcium sensors; in vivo imaging; adaptive thresholding;

$\mathrm{ROI}$ segmentation; automated image analysis; wide-field imaging; two-photon imaging; neural network

\section{Significance Statement}

ACSAT aims at automatically segmenting cells in large-scale calcium imaging datasets. It is based on adaptive thresholding at both global and local levels and iteratively identifies individual neurons in a time-collapsed image. It is designed to address a variety of datasets, potentially involving variations in cell morphology and fluorescence intensity between different datasets. We demonstrate the effectiveness of ACSAT by testing it under a variety of conditions. For the simulated datasets with truth, ACSAT achieved recall and precision rates $>80 \%$ when the signal-to-noise ratio was no less than $\sim 24 \mathrm{~dB}$. For the datasets from mouse hippocampus and striatum, ACSAT captured $\sim 80 \%$ of human-identified ROls and even detected some low-intensity neurons that were initially undetected by human referees.

Received February 3, 2018; accepted August 23, 2018; First published September 04, 2018.

The authors declare no competing financial interests.
Author contributions: S.P.S. designed research, performed research, contributed unpublished reagents/analytic tools, analyzed data, and wrote the paper; H.T. performed research, analyzed data, and wrote the paper; K.R.H. 


\section{Introduction}

The ability to record from a large population of single neurons during behavior greatly facilitates the investigation of the contribution of individual neurons to neuronal network dynamics. Extracellular single-unit recording has traditionally been a method of choice in neurophysiological analyses of single neurons in the brain. Recent improvements, such as the new generation of genetically encoded calcium sensors GCaMP6 (Chen et al., 2013, Sun et al., 2013), have made it possible to observe hundreds to thousands of individual neurons simultaneously (Ohki et al., 2005; Andermann et al., 2010; Huber et al., 2012; Ziv et al., 2013; Issa et al., 2014; Mohammed et al., 2016). Though indirect, these calcium indicators have been sensitive enough to monitor neuronal activity with high spatiotemporal precision in behaving animals, allowing researchers to examine the activity of populations of a specific cell type (Hofer et al., 2011; Wachowiak et al., 2013; Pinto and Dan, 2015; Allen et al., 2017) or the same cell over an extended period of time (Poort et al., 2015).

As the performance of genetically encoded calcium indicators has improved, wide-field microscopy has become feasible for recording the activity of a large population of neurons over an extended anatomical area (Lütcke et al., 2013; Wilt et al., 2013). Although lacking the spatial subcellular resolution of a multiphoton microscope, wide-field microscopes can operate at a higher speed, allowing simultaneous recording of increasingly larger populations (Ghosh et al., 2011; Ziv et al., 2013; Kim et al., 2016; Mohammed et al., 2016). Advanced microfabrication techniques further miniaturized the widefield microscope to a microendoscope capable of monitoring neural activity in freely-moving animals (Ghosh et al., 2011; Ziv et al., 2013).

An emerging technical challenge that parallels advances in calcium imaging is the processing of large datasets (Hamel et al., 2015). During data analysis, an important step is to identify regions of interest (ROIs) corresponding to individual neurons. As data grows rapidly both spatially and temporally, the traditional laborintensive approach of manual inspection has to be automated. Principal component analysis (PCA) and independent component analysis (ICA) methods are natural and frequently used candidates for automating ROI iden-

performed research; R.W. analyzed data; H.G. performed research; J.S. and X.H. designed research and wrote the paper.

X.H. acknowledges funding from National Institutes of Health Director's new innovator award (1DP2NS082126), Pew Foundation, DARPA Young Faculty Award, National Science Foundation (EEC-1647837), Boston University Biomedical Engineering Department. K.R.H. is supported by a National Science Foundation Graduate Research Fellowship (DGE-1247312).

${ }^{*}$ S.P.S. and H.T. are co-first authors.

Correspondence to either of the following: Jennie Si, Arizona State University Goldwater Center \#351, 650 E Tyler Mall, Tempe, AZ85287. E-mail: si@asu.edu; or Xue Han, Boston University, 44 Cummington Mall, Room 401, Boston, MA 02215. E-mail: xuehan@bu.edu.

https://doi.org/10.1523/ENEURO.0056-18.2018

Copyright (C) 2018 Shen et al.

This is an open-access article distributed under the terms of the Creative Commons Attribution 4.0 International license, which permits unrestricted use, distribution and reproduction in any medium provided that the original work is properly attributed. tification (Mukamel et al., 2009). However, if its assumption of statistical independence between neurons is violated, which is often the case in real neural recordings, then the method relies on user selection of parameters for spatial segmentation.

Threshold-based methods represent a promising and intuitive alternative for automatic ROI identification. However, several challenges need to be overcome, including variability in recording conditions or fluorescence signal strength across structures, recording subjects, and the imaging field. For example, one of the most referenced thresholding methods, Otsu's method, which automatically selects the optimal threshold value that minimizes the intraclass variance among $\mathrm{ROI}$ pixels and among background pixels, would only successfully segment some of the highest-intensity ROls (Otsu, 1979; Sezgin and Sankur, 2004). Additionally, the multiclass Otsu's method is limited because uneven lighting may result in separate background classes. A waterfall-thresholding approach addresses uneven lighting by iterative thresholding to capture all intensity peaks, but its selection of a threshold value is ad hoc, making it dataset-dependent and user-dependent (Mellen and Tuong, 2009). A feedback loop-based approach for segmenting bacteria cells optimizes the threshold value from the distribution of pixel intensities, but its assumption that the total ROI area remains constant over time does not hold for calciumimaging datasets because neurons change in brightness (Shen et al., 2015). A recent machine learning-based algorithm uses image gradients and pixel traces to optimize threshold values, but it still requires a user's subjective input in selecting a background removal factor based on each dataset (Fantuzzo et al., 2017). Other approaches based on edge detection have trouble due to weak fluorescence signal strength in comparison with the background pixels (Sadeghian et al., 2009). Generally, most segmentation methods require a high level of tuning to each individual dataset.

To overcome these challenges of diverse imaging datasets, we introduce a new automated cell segmentation by adaptive thresholding (ACSAT) algorithm. ACSAT dynamically and automatically determines global and local threshold values based on the distribution of pixel intensities within a time-collapsed image of a recorded image sequence. We demonstrate the utility of ACSAT on simulated datasets, cell culture datasets, and in vivo widefield and two-photon datasets. For the simulated datasets with truth, ACSAT achieved $>80 \%$ recall and precision when the signal-to-noise ratio was no less than $\sim 24 \mathrm{~dB}$. ACSAT also captured $\sim 80 \%$ of human-identified ROls in datasets from mouse hippocampus and striatum and was even able to detect low-intensity neurons that were initially undetected by human referees.

\section{Materials and Methods}

\section{Wide-field hippocampus and striatum datasets}

All animal procedures were approved by [Boston University] Institutional Animal Care and Use Committee. Female C57BL/6 mice (8-12 weeks old, Taconic) were first injected with $250 \mathrm{~nL}$ AAV9-Syn-GCaMP6.WPRE. 
SV40 virus (titer: $\sim 6 \mathrm{e} 12 \mathrm{GC} / \mathrm{mL}$, University of Pennsylvania Vector Core). AAV was delivered either into the dorsal CA1 (AP: -2, ML: 1.4, DV: -1.6), or into the dorsal striatum (AP: 0.5, ML: 1.8, DV: -1.6$)$ regions. Injections were performed with a $10-\mu \mathrm{L}$ syringe (World Precision Instruments) coupled with a 33-gauge needle (NF33BL, World Precision Instruments) at a speed of $40 \mathrm{~nL} / \mathrm{min}$, controlled by a microsyringe pump (UltraMicroPump 3-4, World Precision Instruments). Upon complete recovery, a custom imaging chamber with glass coverslip was surgically implanted on top of the viral injection site by removing the overlying cortical tissue. The imaging chamber was assembled by fitting a circular coverslip (size 0; OD: $3 \mathrm{~mm}$ ) to a stainless steel cannula (OD: $3.17 \mathrm{~mm}$, ID: $2.36 \mathrm{~mm}$ ) using a UV-curable optical adhesive (Norland Products). During surgery, a custom aluminum headplate was also attached to the skull, which allowed head fixation during the imaging session.

Imaging data were acquired with a custom wide-field microscope coupled with a scientific CMOS camera (ORCA-Flash 4.0, C11440-42U, Hamamatsu), controlled by the commercial software package HClmageLive (Hamamatsu). The wide-field microscope consisted of a Leica N Plan 10× $0.25 \mathrm{PH} 1$ objective lens, an excitation filter (HQ 470/50), a dichroic mirror (FF506-Di02), an emission filter (FF01-536/40), a commercial SLR lens as the tube lens (Nikon Zoom-NIKKOR 80-200 mm f/4 Al-s), and a 5W LED (LZ1-00B200, $460 \mathrm{~nm}$; LedEngin). Data acquisition was performed at $20 \mathrm{~Hz}$, at a resolution of $1024 \times$ 1024 pixels, with 16 bits per pixel, for $\sim 10-20$ min. With $10 \times$ objective lens, the microscope provided a field of view of $1.343 \times 1.343 \mathrm{~mm}^{2}\left(1.312 \times 1.312 \mu \mathrm{m}^{2} /\right.$ pixel $)$ of brain tissue. Imaging data were streamed from the camera to RAM of a custom computer (dual Intel Xeon processors, 128 GB RAM, and a GeForce GTX Titan video card) to ensure temporal precision. After each imaging session, data were moved from RAM to hard drive and saved in multipage tagged image file format.

Two hippocampus datasets (A and $B$ ) were collected from two mice [dataset $A$ was previously reported by Mohammed et al. (2016)]. The mice were trained to perform a trace conditioning task known to involve hippocampal neural activity (Solomon et al., 1986; Moyer et al., 1990; Tseng et al., 2004; Sakamoto et al., 2005). In this task, the animal was trained to associate a conditioned stimulus (a 350-ms-long tone) with an unconditioned stimulus (a gentle 100-ms air puff to one eye). There was a 250-ms trace interval between two stimuli. During each recording session, the animal was head-fixed and performed 40 trials with a randomized 31-36-s intertrial interval. The hippocampus datasets $(1024 \times 1024$ pixels/frame, 2047 frames, $\sim 100 \mathrm{~s}, \sim 4$ GB size) analyzed in this study were part of larger recording sessions $(\sim 50$ GB size).

The striatum dataset was collected from a head-fixed animal running on a spherical treadmill system. The treadmill system consisted of a Styrofoam ball floated by air pressure in a 3D-printed bowl designed as described in Dombeck et al. (2007) that allowed the animal to move its limbs freely while head-fixed. The mouse was first han- dled for several days before being head-fixed to the spherical treadmill. Habituation to running on the spherical treadmill while head-fixed occurred over 3-4 days/ week at the same time of day as subsequent recording sessions (8-12 h after lights-on), for several weeks. Single imaging sessions took $\sim 25 \mathrm{~min}$. Sampling occurred at $\sim 20 \mathrm{~Hz}$, and exposure time was fixed at $20 \mathrm{~ms}$. The striatum dataset $(\sim 100 \mathrm{~s}, \sim 4 \mathrm{~GB}$ size) contains 2047 frames with $1024 \times 1024$ pixels per frame and was also part of a larger dataset ( $\sim 25$ GB size).

Two human referees manually identified ROls in the hippocampus dataset $A$ and in the striatum dataset to create a set of human-generated ROls for comparison with ACSAT's segmentation results. This manual selection was done by viewing the image sequence and segmenting ROls that had fluorescence traces compatible with neuronal dynamics and/or by selecting ROls from a composite image created from the video sequence and confirming that fluorescence traces were compatible with neuronal dynamics.

\section{Wide-field cell culture dataset}

The primary neuron cell dataset was collected from a 10-day-old neuron culture, infected with AAV9-SynGCaMP6.WPRE.SV40 virus. Seven days after infection, neurons were imaged at $20 \mathrm{~Hz}$ for $60 \mathrm{~s}$. The primary neuron culture dataset contains 1201 frames, $1024 \times$ 1024 pixels per frame, recorded with the same imaging setup as for the hippocampus and striatum datasets described above.

\section{Two-photon dataset}

The two-photon dataset was downloaded from the Neurofinder website (http://neurofinder.codeneuro.org/, 03.00). GCaMP6f was used as the indicator. The dataset contains 2250 frames with $498 \times 490$ pixels per frame with resolution $0.588 \times 0.588 \mu \mathrm{m}^{2} /$ pixel.

\section{Signal-to-noise ratio (SNR) calculation}

We calculated the SNR in decibels $(\mathrm{dB})$ as

$$
\mathrm{SNR}=20 \times \log \frac{\mu_{\mathrm{ROI}}}{\sigma_{\text {background }}} .
$$

For the simulated datasets, $\mu_{\mathrm{RO}}$ is the mean intensity value of all pixels belonging to all ROls in the timecollapsed image $I_{0}$, and similarly, $\sigma_{\text {background }}$ is the standard deviation of background pixel intensity values, i.e., all pixels that do not belong to an ROI. For the hippocampus dataset $\mathrm{A}$ and the striatum dataset, $\mu_{\mathrm{RO}}$ is the maximumintensity value of an ROI trace, and $\sigma_{\text {background }}$ is the standard deviation of the background trace. The ROI trace value at each time point is the averaged intensity values of all pixels belonging to that ROI, and similarly for the background trace, which uses all pixels not belonging to any ROI. Note that the SNR for the simulated datasets describes the whole time-collapsed image $I_{0}$, whereas the SNRs for the hippocampus and striatum datasets describe an individual ROI. 
Automated Cell Segmentation by Adaptive Thresholding (ACSAT)

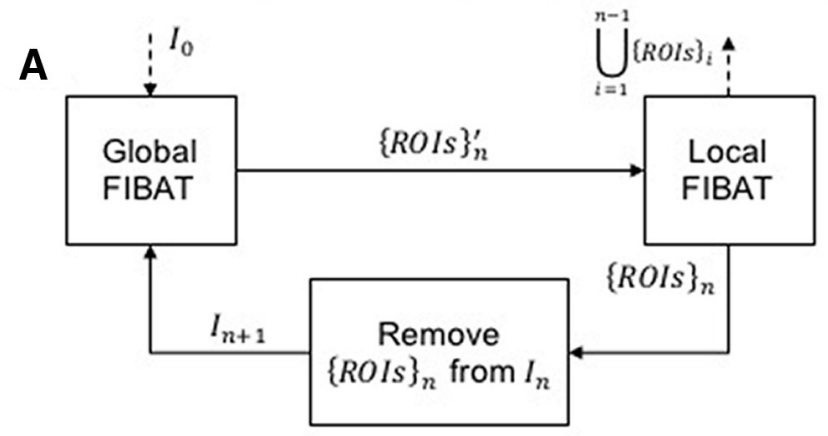

Fluorescence Intensity Based Adaptive Thresholding (FIBAT)
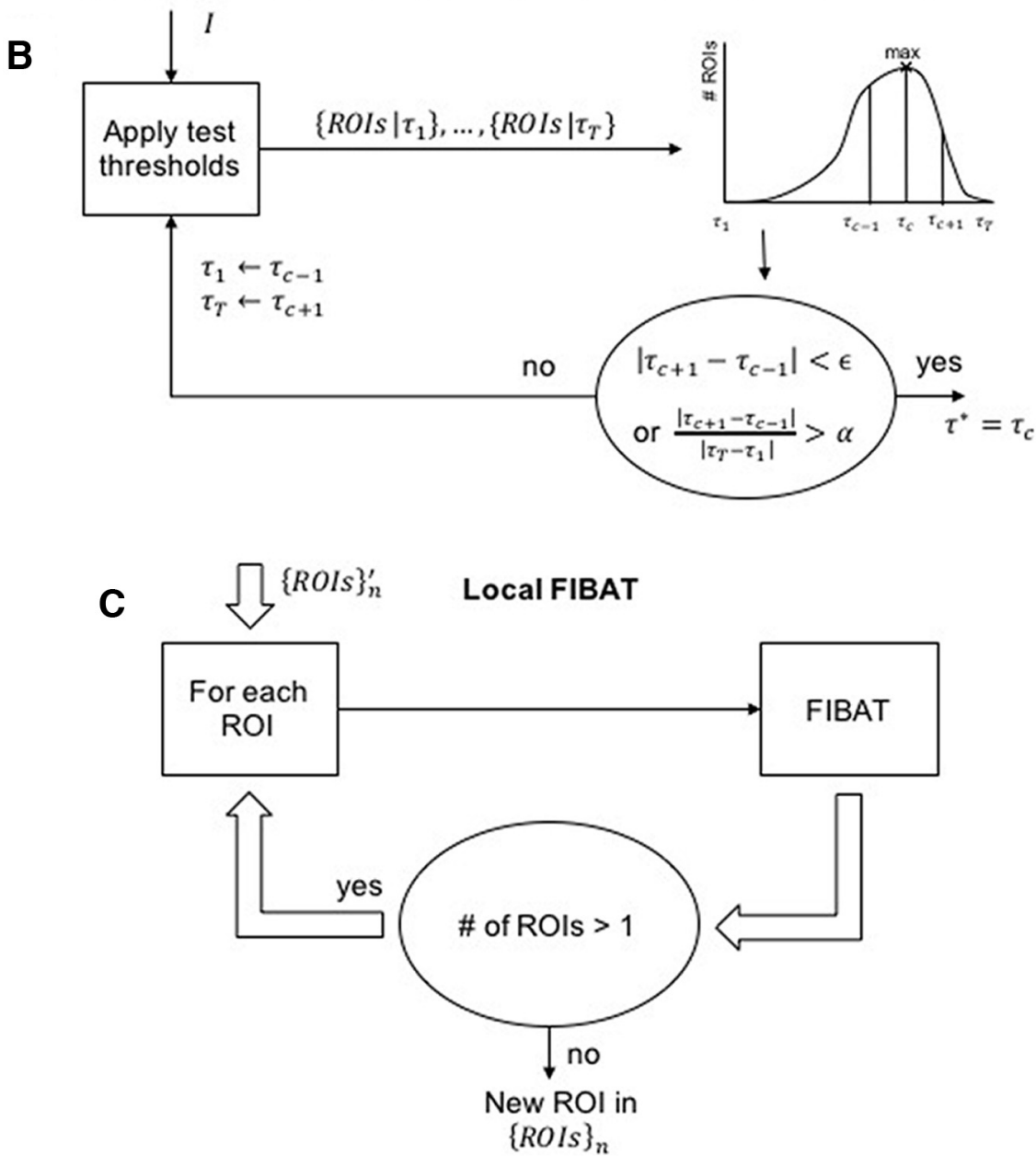

Figure 1. Flowchart of the ACSAT algorithm. $\boldsymbol{A}$, The input is the time-collapsed image $I_{0}$, and the output is a collection of automatically segmented ROls. In each iteration, the Global FIBAT step identifies potential ROIs $\{R O / s\}_{n}^{\prime}$ by applying FIBAT, described in $\boldsymbol{B}$, to the entire image $I_{n}$; and the Local FIBAT step, described in $\boldsymbol{C}$, splits overlapping ROIs. $\boldsymbol{B}$, Flowchart of the FIBAT algorithm. The input image is segmented using each of the test threshold values $\tau_{1}, \tau_{2}, \ldots, \tau_{T}$. The search range for the optimal threshold value $\left(\tau_{1}, \tau_{T}\right)$ is iteratively narrowed to contain the test threshold value which results in the maximum number of ROls. $\boldsymbol{C}$, Local FIBAT procedure. FIBAT, described in $\boldsymbol{B}$, is recursively applied to each potential ROI until the resulting ROls can no longer be separated by FIBAT.

\section{Simulated datasets}

We tested ACSAT's segmentation performance on 500 simulated datasets with varying SNRs (between $\sim 19$ and $\sim 29 \mathrm{~dB}$ ) and numbers of ROls (between 300 and 700). Fig.
$2 B$ shows some examples of the simulated time-collapsed image, i.e., the input image $I_{0}$ to ACSAT in Fig. $1 A$. The simulation gives us the true locations of all ROls so that we can accurately assess ACSAT's segmentation performance. 
Our simulated datasets were obtained by a procedure adapted from Zhou et al. (2018). We used the model $I_{0}=E+A C$ to generate the simulated datasets, where $E$ represents noise, $A$ represents the shapes of each ROI, and $C$ adjusts each ROl's intensity to simulate uneven lighting.

The pixel noise values in $E$ were randomly sampled from the background pixel values in the time-collapsed image for the hippocampus dataset. This noise is unlikely to be Gaussian, because the time-collapsing procedure subtracts the mean value from the maximum value of each pixel such that the time-collapsed image is biased toward higher pixel values.

The centroid location of each $\mathrm{ROI}$ represented in $A$ was randomly selected with weights $C^{2}$. The pixel values comprising the body of each $\mathrm{ROI}$ was modeled deterministically by the bivariate Gaussian probability density function, with widths randomly selected according to Zhou et al. (2018).

The image $C$ is also used to amplify each ROl's pixel values to reflect uneven lighting conditions across the imaging field. $C$ was generated by applying heavy Gaussian filtering to the time-collapsed image of the hippocampus dataset until no individual ROls are detectable.

\section{ACSAT overview}

Fluorescence imaging data obtained in the form of image sequences is processed offline using a custom Matlab algorithm. Image sequences were first motioncorrected as described in Mohammed et al. (2016) to remove micromotion of the imaged area caused by breathing and other movements of the animal. ACSAT (Fig. $1 A$ ) is then applied to a time-collapsed image that represents the image sequences, to automatically identify individual neurons as ROls.

The input image sequence is first loaded into Matlab as a 3D matrix (height $\times$ width $\times$ time) and then timecollapsed to produce a representative two-dimensional image (height $\times$ width, $I_{0}$ in Fig. $1 A$ ), where each pixel in $I_{0}$ is represented by the maximum-intensity value of that pixel across the entire image sequence with its mean value removed. This time-collapsed image $I_{0}$ is then used for the rest of the algorithm. Pixels with low-intensity values would correspond to static background, whereas pixels with high-intensity values would correspond to neurons with GCaMP6 expression. In general, neurons with GCaMP6 expression appear in $I_{0}$ as a cluster of adjacent pixels with high-intensity values and with size similar to that of a neuron. Meanwhile, it is improbable for random background noise to generate clusters with similar properties. Thus, the time-collapsed image $I_{0}$ is expected to contain sufficient information to separate neurons from the background.

Next, ACSAT iteratively generates ROls $\{R O / s\}_{n}$ from the time-collapsed image $I_{n}$ for iterations $n=1,2, \ldots$, starting with $I_{1}=I_{0}$. Before each subsequent iteration, $I_{n}$ is generated by cumulatively clearing previously segmented ROls, $\{R O / s\}_{n-1}$, from $I_{n-1}$ by setting each ROl's pixels in $I_{n-1}$ to blank values of 0 and dilating the cleared area. As described later, each iteration consists of both adaptive thresholding at the global level (Global FIBAT in Fig. 1A), using the automatically selected threshold value $\tau_{n}^{*}$ (Fig. $1 B$ ), and adaptive thresholding at the local level (Local FIBAT in Fig. 1A). When the change in global threshold value $\left|\tau_{n+1}^{*}-\tau_{n}^{*}\right|$ is insignificant, further iterations are likely to contribute more false positives than true positives. Thus, the ACSAT algorithm terminates at iteration $n$ if

$$
\frac{\left|\tau_{n+1}^{*}-\tau_{n}^{*}\right|}{\tau_{1}^{*}}<\delta,
$$

where $\tau_{1}^{*}$ acts as a normalizing factor. Accordingly, the final output of ACSAT is the union of the segmented ROls from each iteration, $\{R O / s\}_{1} \cup \cdots \cup\{R O / s\}_{n}$.

\section{Global and local adaptive thresholding in ACSAT}

Each iteration $n$ of ACSAT contributes a set of newly segmented ROls $\{R O / s\}_{n}$ from $I_{n}$ by applying our fluorescence intensity based adaptive thresholding (FIBAT) algorithm, at the global and local levels (Global/Local FIBAT in Fig. 1A). Briefly, FIBAT (Fig. 1B) takes an inputted image $I$ and outputs the optimal threshold value $\tau^{*}$ which results in optimally segmented ROIs $\left\{R O / s \mid \tau^{*}\right\}$.

Global adaptive thresholding is the first step in the $n$th iteration of ACSAT (Fig. 1A). This step applies FIBAT directly to the whole image $\left(I_{n} \rightarrow I\right)$ to identify potential ROIs $\left(\left\{R O / s \mid \tau^{*}\right\} \rightarrow\{R O / s\}_{n}^{\prime}\right)$.

These potential ROIs $\{R O / s\}_{n}^{\prime}$ may include groups of adjacent neurons or overlapping neurons because neurons located above and below the focal plane could be captured in the same frame during wide-field imaging. Such overlap, however, is unlikely to occur in two-photon datasets or in cell culture datasets. The local adaptive thresholding step (Fig. $1 C$ ) recursively separates any potentially overlapping ROls within $\{R O / s\}_{n}^{\prime}$ to output $\{R O / s\}_{n}$. Specifically, each $\mathrm{ROI}$ in $\{R O / s\}_{n}^{\prime}$ is individually dilated and then inputted to the local FIBAT (ROI $\rightarrow I)$ in Fig. $1 B$ to obtain a set of separated ROls $\left\{R O / s \mid \tau^{*}\right\}$. If any outputted set $\left\{R O I s \mid \tau^{*}\right\}$ contains more than one separated ROI, then each $\mathrm{ROI}$ in the set $\left\{R O / s \mid \tau^{*}\right\}$ is further separated using the same procedure, thus forming a recursive loop. Otherwise, if any outputted set $\left\{R O / s \mid \tau^{*}\right\}$ contains only one $\mathrm{ROI}$, then the recursion terminates. The final output of the local thresholding step $\{R O / s\}_{n}$ is the union of all such sets containing one $\mathrm{ROI}$ that cannot be further separated.

\section{FIBAT}

As described, FIBAT (Fig. 1B) is used in both the global and local adaptive thresholding steps of each iteration of ACSAT to identify potential ROls in the time-collapsed image $I=I_{n}$ or to separate potentially overlapping neurons within I which is an element of $\{R O / s\}_{n}^{\prime}$, respectively. In either case, an optimal pixel intensity threshold value $\tau^{*}$ separates ROls from the background. FIBAT selects $\tau^{*}$ by searching for the threshold value that maximizes the number of resulting ROls that are larger in area than $A_{\min }$ and smaller in area than $A_{\max }$.

The search is performed recursively over a pixel intensity range $\left(\tau_{1}, \tau_{T}\right)$, where initially $\tau_{1}$ is the minimum pixel intensity value in $I$ and $\tau_{T}$ is the maximum pixel intensity value in $I$. From this search range, $T$ test threshold values 
$\tau_{1}, \tau_{2}, \ldots, \tau_{T-1}, \tau_{T}$ are uniformly selected. A larger $T$ will decrease the probability of skipping the optimal threshold value, but it will result in more computation time that may not be necessary. Because the threshold value is refined by a recursive process until it reaches the optimal value that produces the maximum number of ROls, the value of $T$ should have little to no effect on ACSAT's segmentation results. We chose $T=12$. Each of these test threshold values $\tau_{1}, \ldots, \tau_{T}$ is applied to the image $/$ by assigning each pixel a 1 (a true calcium event) if its value is greater than the threshold or a 0 (a false calcium event) otherwise. Morphological operations are then performed to refine the thresholded images. Specifically, these operations fill in holes (0s surrounded by 1s) and remove spur pixels that may be due to noise. The operations also break $\mathrm{H}$-connected ROls before splitting overlapping cells. ROls are finally collected with 8-connectivity (Matlab function bwlabel or bwconncomp) to generate a set of segmented ROls for each test threshold value: $\left\{R O / s \mid \tau_{1}\right\}, \ldots,\left\{R O / s \mid \tau_{T}\right\}$.

Since ROls represent real neurons that are roughly spherical in shape and are $\sim 5-20 \mu \mathrm{m}$ in diameter, some realistic criteria can be used to eliminate false ROls that are not possibly actual neurons. Accordingly, FIBAT removes ROls from $\left\{R O / s \mid \tau_{1}\right\}, \ldots,\left\{R O / s \mid \tau_{T}\right\}$ if their centroid is outside the $\mathrm{ROI}$, or if their area is less than $A_{\min }$ or greater than $A_{\max }$, or if their solidity (i.e., the area ratio between the convex hull of a ROI and the ROI itself) is greater than approximately the golden ratio.

The next search range is selected based on the results of the test thresholds. A relationship of the test threshold values $\tau_{1}, \ldots, \tau_{T}$ versus the numbers of resulting ROIs $\left|\left\{R O / s \mid \tau_{1}\right\}\right|, \ldots,\left|\left\{R O / s \mid \tau_{T}\right\}\right|$ can be generated (Fig. 1B). If the test threshold value $\tau_{c}$ resulted in the most ROls, i.e., $c=\operatorname{argmax}_{c}\left|\left\{R O / s \mid \tau_{c}\right\}\right|$, then the next search range is set to $\left(\tau_{\max \{1, c-1\}}, \tau_{\min \{T, c+1\}}\right)$ to include $\tau_{c}$ inside the search range. If more than one test threshold value $\tau_{c_{1}}, \tau_{c_{2}}, \ldots$ resulted in the same maximum number of ROIs, then the next search range is similarly set to $\left(\tau_{\max \left\{1, \min \left\{c_{1}, c_{2}, \ldots\right\}-1\right\}}, \tau_{\min \left\{T, \max \left\{c_{1}, c_{2}, \ldots\right\}+1\right\}}\right)$ to contain all $\tau_{c_{1}}, \tau_{c_{2}}, \ldots$. This search is terminated when further refinement of the search range produces little improvement in the number of detected ROls: either the new search range $\mid \tau_{c+1}-$ $\tau_{c-1} \mid$ is less than $\varepsilon$ or the new range overlaps the previous range by at least $\alpha$. We chose $\alpha=90 \%$ and $\varepsilon=$ the smallest nonzero intensity difference between every pair of adjacent pixels in whole image $I$. As such, $\varepsilon$ is determined automatically and does not require user input. On termination, the optimal threshold value is set to $\tau^{*}=$ $1 / 2\left(\min \left\{\tau_{c_{1}}, \tau_{c_{2}}, \ldots\right\}+\max \left\{\tau_{c_{1}}, \tau_{c}, \ldots\right\}\right)$, and the segmented ROIs $\left\{R O / s \mid \tau^{*}\right\}$ includes ROls whose area exceeds $A_{\max }$.

\section{Code accessibility}

The code/software described in the paper is freely available online at https://github.com/sshen8/acsat. The code is available in Extended Data 1.

\section{Results}

We tested ACSAT on 500 simulated datasets, two wide-field hippocampus datasets, a wide-field striatum dataset, a wide-field cell culture dataset, and a twophoton hippocampus dataset. The simulated datasets with known ground truth allowed us to accurately assess the segmentation performance of ACSAT in different conditions of SNR and number of ROIs. For the hippocampus dataset $A$ and the striatum dataset, in which the ground truth is unknown, we used human-generated ROls as a reference. For the cell culture dataset, hippocampus dataset $\mathrm{B}$, and two-photon dataset, we provide the ACSAT segmented ROls that can be inspected and interpreted by users.

\section{ACSAT performance on simulated datasets with various SNRs and numbers of ROls}

To evaluate the performance of ACSAT, we simulated 500 time-collapsed images $I_{0}$ with various numbers of ROls (between 300 and 700 ) at random locations and different SNRs (between $\sim 19$ and $\sim 29 \mathrm{~dB}$ ). The exact locations of ROls are known and served as the ground truth to provide an accurate evaluation of the performance of ACSAT. For all 500 datasets, we used the parameters $\delta=10 \%, A_{\min }=50 \mathrm{px} \approx 86 \mu \mathrm{m}^{2}$ and $A_{\max }=300 \mathrm{px} \approx$ $516 \mu \mathrm{m}^{2}$ for the global adaptive thresholding step, and $A_{\min }=20 \mathrm{px} \approx 34 \mu \mathrm{m}^{2}$ and $A_{\max }=\infty$ for the local adaptive thresholding step because ROls tend to shrink in size after repeatedly applying FIBAT.

The recall and precision results for each of these simulated datasets are shown as dots in Fig. $2 A 1$ and Fig. $2 A 2$, respectively. Fig. $2 B$ shows examples of the simulated time-collapsed images, and each example corresponds to a dot in Fig. 2A1 and Fig. 2A2. At SNR greater than $\sim 24 \mathrm{~dB}$, ACSAT shows a stable performance with generally higher than $80 \%$ recall. The precision rate remains stable at generally higher than $80 \%$ when SNR is greater than $\sim 21 \mathrm{~dB}$. However, the performance of ACSAT falls when SNR is below $\sim 20 \mathrm{~dB}$.

\section{ACSAT performance on hippocampus dataset $A$ and striatum dataset}

We used ACSAT (Fig. 1A) to automatically segment ROls from a hippocampus wide-field imaging dataset and a striatum wide-field imaging dataset. Before the application of the ACSAT, the image sequences were timecollapsed as shown in Figs. 3 and 4 (top rows) for the hippocampus $A$ and the striatum datasets, respectively. These time-collapsed images show high-intensity areas resembling neural morphology. The final segmented ROIs outputted by ACSAT are illustrated in Figs. 3 and 4 (bottom row), respectively.

For both datasets, we initiated ACSAT using the same parameters as for the simulated datasets $(\delta=10 \%$, $A_{\min }=50 \mathrm{px} \approx 86 \mu \mathrm{m}^{2}$ and $A_{\max }=300 \mathrm{px} \approx 516 \mu \mathrm{m}^{2}$ for the global adaptive thresholding step, and $A_{\min }=$ $20 \mathrm{px} \approx 34 \mu \mathrm{m}^{2}$ and $A_{\max }=\infty$ for the local adaptive thresholding step). To obtain the results as shown in Figs. 3 and 4, it took approximately $1 \mathrm{~min}$ per iteration on a Xeon E5-1650 v4 at 3.6 GHz with 128 GB DDR4 RAM, but it used $<30$ MB RAM. As such, the RAM size had little effect on the speed.

ACSAT performance compared to human-generated ROIs

To assess the performance of the ACSAT algorithm, we compared the ACSAT segmentation results with ROIs 
A1

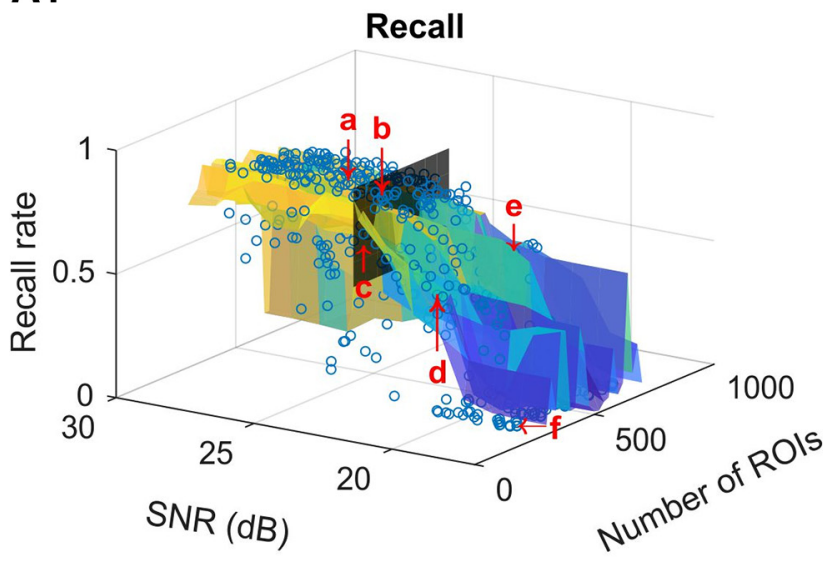

\section{A2}

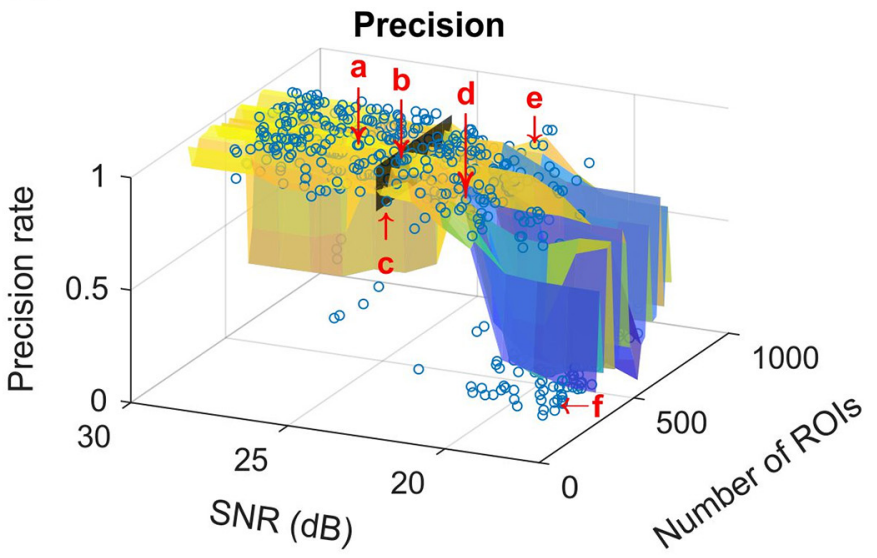

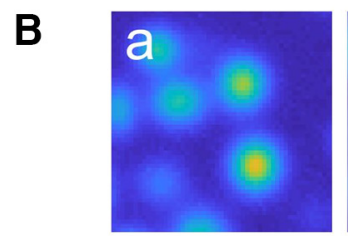
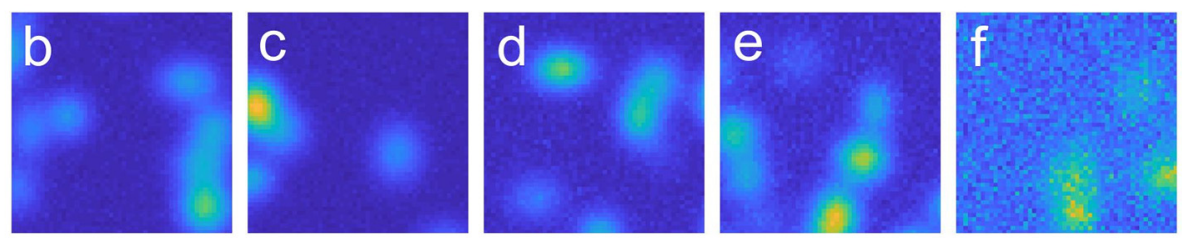

\section{SNR=25.3487 SNR=23.7261 SNR=23.7088 SNR=21.521 SNR=20.7313 SNR=18.4537}

Figure 2. ACSAT performance on simulated datasets. $\boldsymbol{A 1}, \boldsymbol{A 2}$, Recall $(\boldsymbol{A 1})$ and precision $(\boldsymbol{A 2})$ are plotted as a function of SNR and number of ROls. Each dot corresponds to the ACSAT result for one of the 500 simulated datasets. A surface was fitted to these dots for visualization. The black vertical plane corresponds to the SNR of the hippocampus A dataset. $\boldsymbol{B}$, Six examples of simulated time-collapsed images, labeled a-f, correspond to the dots labeled a-f in $\boldsymbol{A 1}$ and $\boldsymbol{A 2}$.

\section{A}

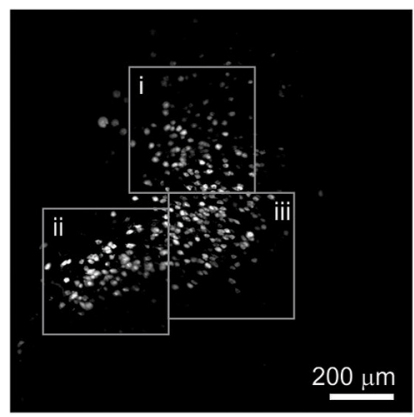

B

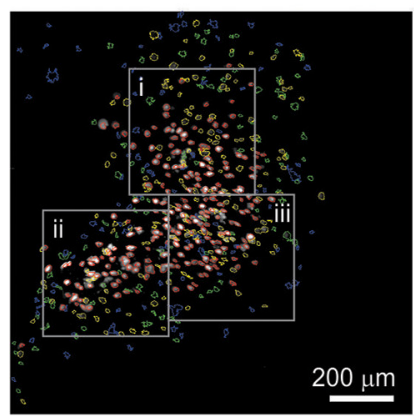

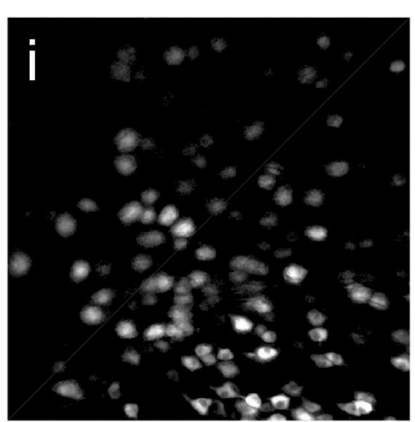
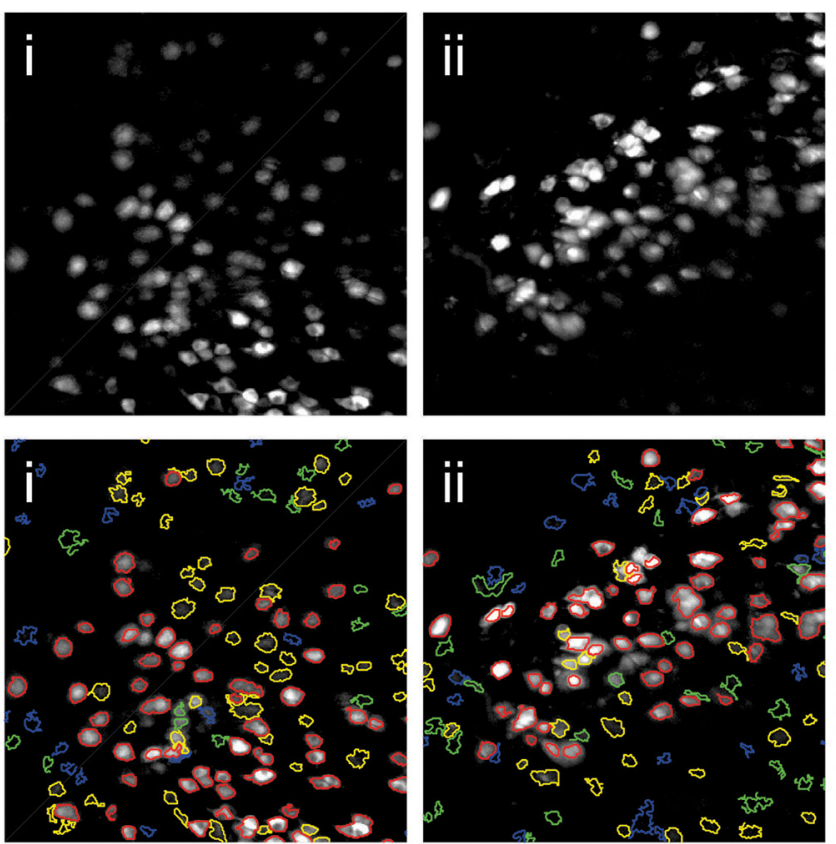
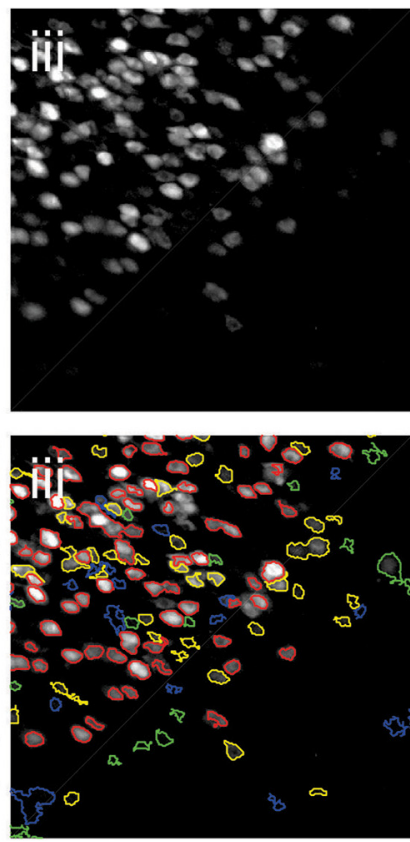

Figure 3. Hippocampus dataset $A$ and ROls identified by ACSAT. $\boldsymbol{A}$, The time-collapsed image of hippocampus dataset $A$ and zoom-in images (Ai, $\boldsymbol{A i i}$, and $\boldsymbol{A i i i}$, corresponding to the gray boxes). $\boldsymbol{B}$, ACSAT-determined ROls from multiple iterations overlying on the time-collapsed image (red, yellow, green, and blue outline corresponds to iteration 1, 2, 3, and 4, respectively). The fourth iteration (blue) is shown for comparison although ACSAT terminated at iteration 3 (red, yellow, and green). 
A

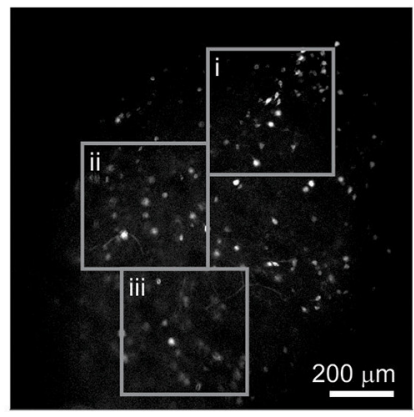

B

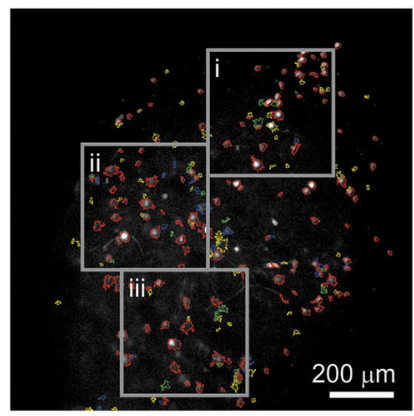

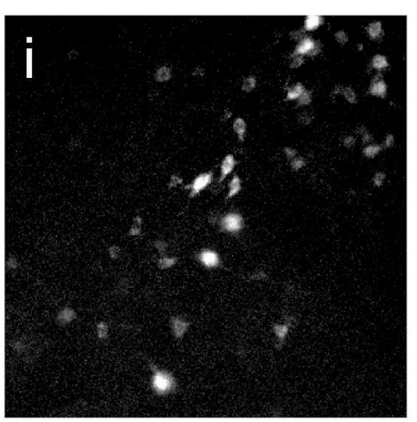
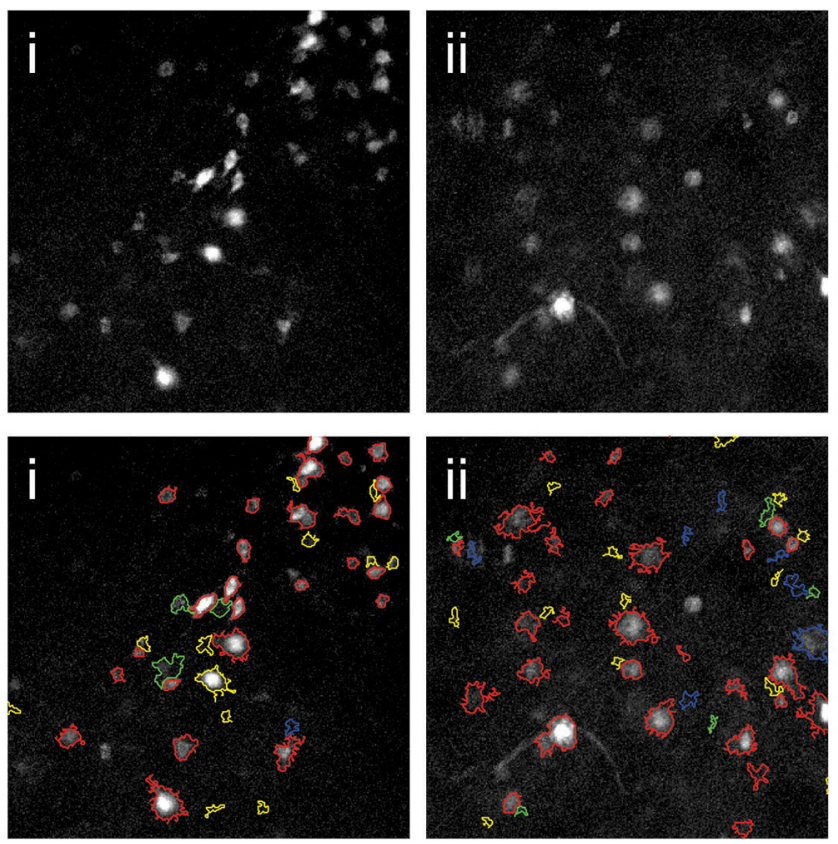
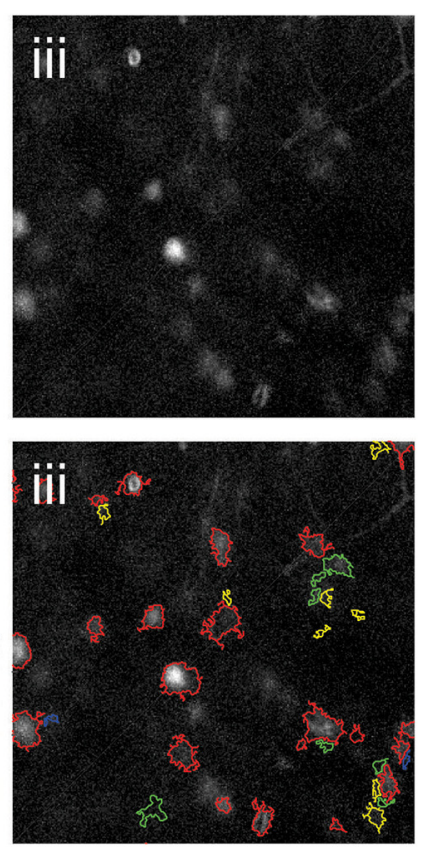

Figure 4. Striatum dataset and ROls identified by ACSAT. $\boldsymbol{A}$, The time-collapsed image of striatum dataset and zoom-in images (Ai, $\boldsymbol{A}$ ii, and $\boldsymbol{A}$ iii, corresponding to the gray boxes). B, ACSAT-determined ROls from multiple iterations overlying on the time-collapsed image (red, yellow, green, and blue outline corresponds to iteration 1, 2, 3, and 4, respectively). The second (yellow), third (green), and fourth (blue) iterations are shown for comparison although ACSAT terminated at iteration 1 (red).

generated by human inspection (human-generated ROIs). This set of human-generated ROls contained 423 ROls for the hippocampus dataset $A$ and 91 ROls for the striatum dataset. We first compared the ACSAT-generated ROIs for the hippocampus $A$ and striatum datasets with the ROls in the human-generated ROls. We consider a pair of ROls to correspond to the same neuron if they had centroids that were $<50 \mathrm{px} \approx 65.6 \mu \mathrm{m}$ apart and had a mutual overlap $>60 \%$. We calculated the mutual overlap as the average of the percentages of the overlapping area against the areas of both ROls. When there were multiple ROls sharing overlapping areas, we selected the pair with highest mutual overlap as the matched ROls.

For the hippocampus dataset A, ACSAT identified 445 ROls after three iterations. Among these 445 ROls, 317 ROls were matched in the human-generated ROls (Match), and 128 ROls were not in the human-generated ROIs (ACSAT-only). Additionally, 106 ROIs in humangenerated ROls were not identified by ACSAT (Humanonly). This result gave us a precision rate of $71.2 \%(317$ out of 445 ) and a recall rate of $74.9 \%$ (317 out of 423 ). For the striatum dataset, ACSAT was terminated after one iteration and identified a total of 135 ROIs: 69 Match ROIs, 66 ACSAT-only ROls, and 22 Human-only ROls (precision rate: $51.1 \%$, recall rate: $75.8 \%$ ).

We further examined the fluorescence traces of ROls from the ACSAT-only, Human-only, and Match groups. Representative traces are shown in Fig. $5 A 1, B 1$, respectively, for the hippocampus $A$ and striatum datasets, and all traces are available in Extended Data 2. The value of each $\mathrm{ROI}$ fluorescence trace at each time point is the average intensity value of all pixels belonging to that $\mathrm{ROI}$. In Fig. $5 A 1, B 1$, each trace is normalized by subtracting the mean value of that trace over time and then dividing the difference by that mean value. We calculated the SNR for every ROI in each group. In both the hippocampus A and striatum datasets, the Match ROIs exhibit a broad range of SNR, indicating that both ACSAT and humans are capable of identifying ROls with various intensities in the time-collapsed image (Fig. 5A2,B2).

We further examined the individual ROls identified by ACSAT that were not identified by humans. This secondary manual inspection found that some of the ACSAT-only ROls were actually true neurons (i.e., with fluorescence traces compatible with neuronal dynamics) that were missed in the initial human-generated ROls because of human error. This means that ACSAT was able to segment ROls that were difficult to identify by human experts. Specifically, for the hippocampus A dataset, 70 (54.7\%) out of 128 ROls initially labeled as ACSAT-only were later determined to be actual neurons, and for the striatum dataset, 31 (47\%) ROls were true neurons. After correction, of the total 445 ACSAT ROls from the hippocampus dataset $A, 387$ segmented ROls corresponded to true neurons (Match), and 58 segmented ROls did not correspond to true neurons determined by human inspection (ACSAT-only). Additionally, 106 true ROls were not segmented (Human-only). This corresponds to a precision rate of $87 \%$ and a recall rate of $78.5 \%$. Similarly, for the striatum dataset, which resulted in 135 ACSAT ROIs, there were 100 Match ROIs, 35 ACSAT-only ROls, and 22 Human-only ROls after correction. This corresponds to a precision rate of $74.1 \%$ and a recall rate of $82 \%$. Although neurons in the hippocampus and striatum have different morphology and fluorescence intensity, ACSAT was consistently effective for both datasets, and it was able to 
A1

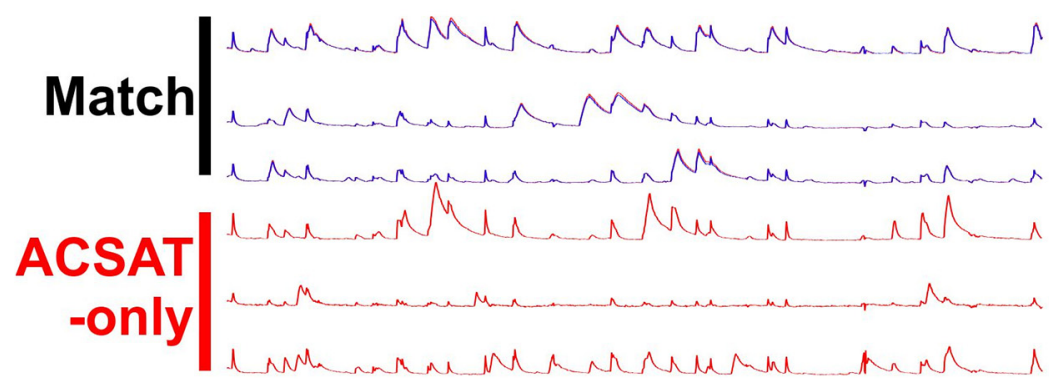

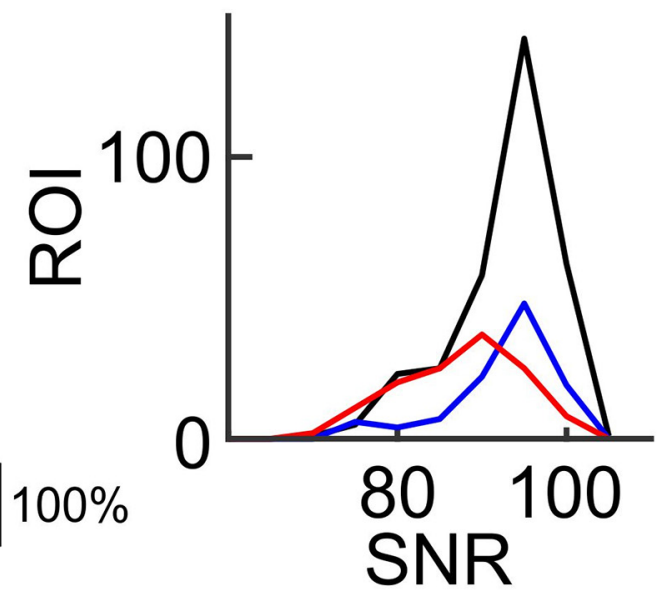

B2
B1

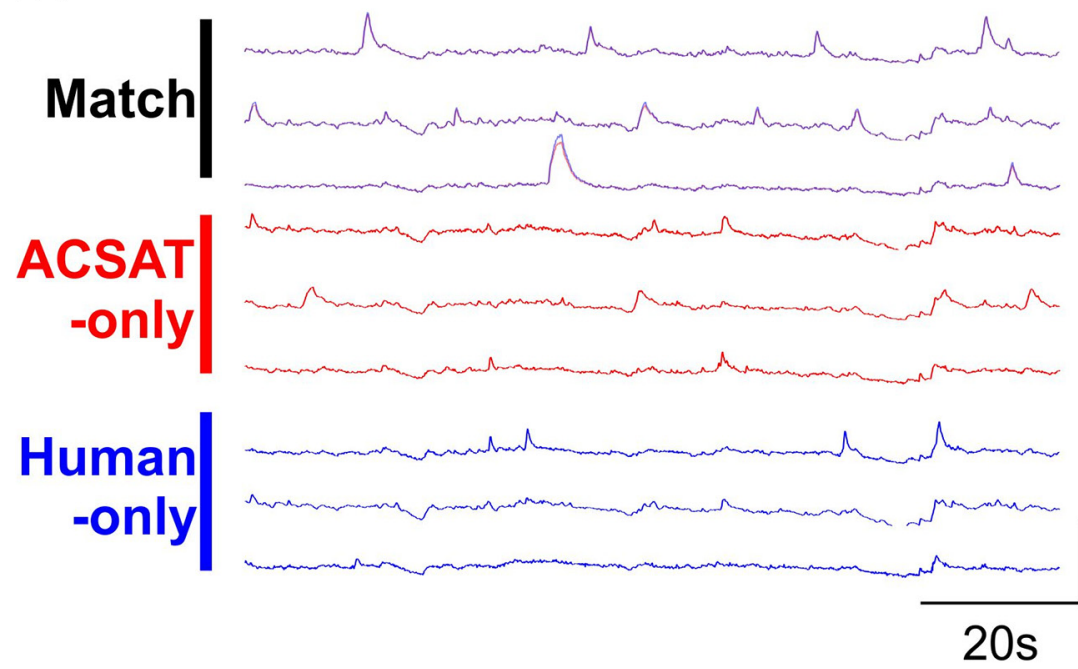

$50 \%$

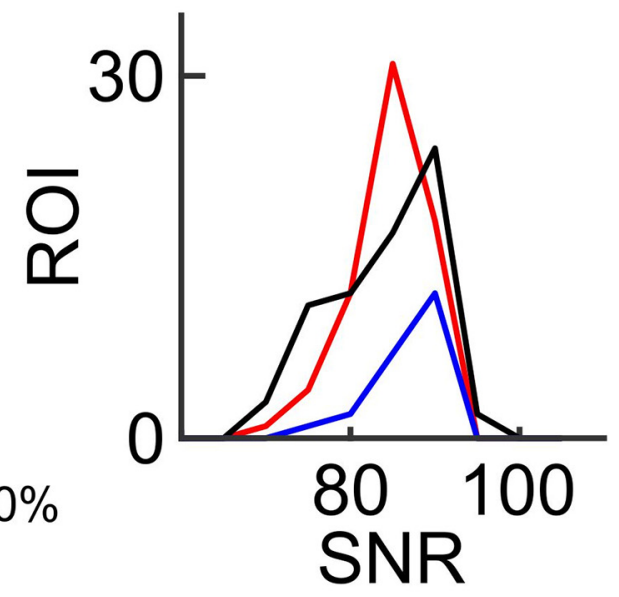

Figure 5. Fluorescence traces and SNRs. A1, Representative fluorescence traces from the hippocampus dataset A for ROIs identified by both ACSAT and human referees (Match), ROls identified only by ACSAT, and ROls identified only by human referees (Human). A2, Histogram of SNR for Match, ACSAT, and human ROls from the hippocampus dataset A. B1, Representative fluorescence traces from the striatum dataset. B2, Histogram of SNR for the striatum dataset.

detect low-intensity neurons that were initially undetected by human referees. As such, our results demonstrate the robustness and effectiveness of the algorithm.

The result from the hippocampus dataset $A$ shows that ACSAT successfully identified true ROls of diverse sizes (Fig. 6, red). In general, the false-positive ROls had relatively smaller areas (Fig. 6, yellow), similar to the ROls missed by human referees (Fig. 6, green). This indicates that ACSAT is more likely to recognize intensity changes in small areas, thereby outperforming human referees under such challenging detection conditions. Additionally, ACSAT missed a small portion of true ROls, which shares similar sizes with those identified (Fig. 6, blue).

Number of iterations in using ACSAT

For the hippocampus dataset A, ACSAT was terminated at iteration $n=3$ when the change in global threshold value

$$
\frac{\left|\tau_{4}^{*}-\tau_{3}^{*}\right|}{\tau_{1}^{*}}=5.12 \%<\delta=10 \%
$$

For the striatum dataset, ACSAT was terminated at iteration $n=1$ when the change in global threshold value

$$
\frac{\left|\tau_{2}^{*}-\tau_{1}^{*}\right|}{\tau_{1}^{*}}=4.53 \%<\delta=10 \%
$$

To evaluate how ACSAT performs when terminated at different iteration numbers, we ran ACSAT up to nine iterations on both datasets, and calculated several major performance indicators after each iteration (Fig. 7): cumulative number of ROls, global threshold value, recall, falsenegative rate, and false discovery rate (which is equal to 1 - precision) compared to the human-generated ROls be- 


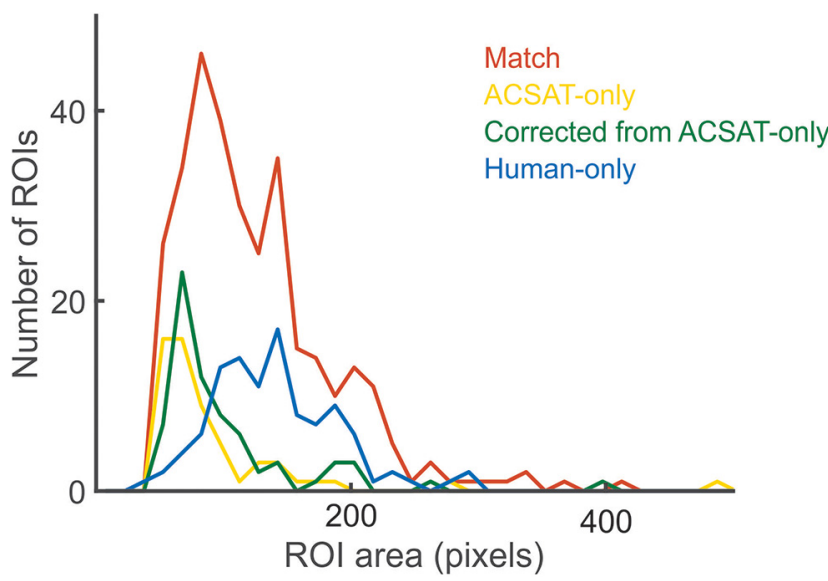

Figure 6. Distribution of ROI size for hippocampus dataset $A$. ROls identified by ACSAT and human (red) with various size. The ACSAT-only ROIs (yellow) and those missed by human experts (green) tend to have small areas, while the areas of human-only ROls (blue) appear slightly larger.

fore secondary manual inspection of false positives. The cumulative number of ROls, recall, and false discovery rate increased with the iteration number, but at different speed. While the cumulative number of ROls and the false discovery rate increased steadily, recall rose steeply and reached its plateau within approximately three iterations for the hippocampus dataset and after the first iteration for the striatum dataset. Both the global threshold value and the false-negative rate dropped as iterations progressed, indicating that ACSAT dynamically adjusted the threshold to capture potential ROIs with lower intensity in later iterations. This dynamic adjustment of the threshold value at each iteration was possible only because of the removal of segmented ROls before each iteration. Overall, the changes in these performance indicators over iterations suggested that most true ROIs were identified during the early iterations: $n \leq 3$ for the hippocampus dataset and $n=1$ for the striatum dataset, which are consistent with when the ACSAT termination criterion described by $\delta$ was met. ROls segmented during later iterations were mostly false positive.

\section{FIBAT global and local thresholding}

In Fig. 8, we demonstrate how FIBAT (Fig. 1B) determines the threshold value that achieves optimal segmentation results by sampling the distribution of threshold values versus the number of ROls. Each trace of Fig. 8 plots the number of ROls that results from each sampled threshold value in the global thresholding step during the first four iterations of ACSAT (Fig. 1A) on the hippocampus dataset $A$. In each iteration, FIBAT (Fig. 1B) first samples the threshold values across the entire intensity range at coarse resolution to identify the potential search range that may result in the maximum number of ROls. FIBAT further resamples threshold values within the new search range with a finer resolution, until it reaches a threshold value that gives the maximum number of ROls. This design allows FIBAT to determine the optimal threshold value with a fine resolution without actually sampling
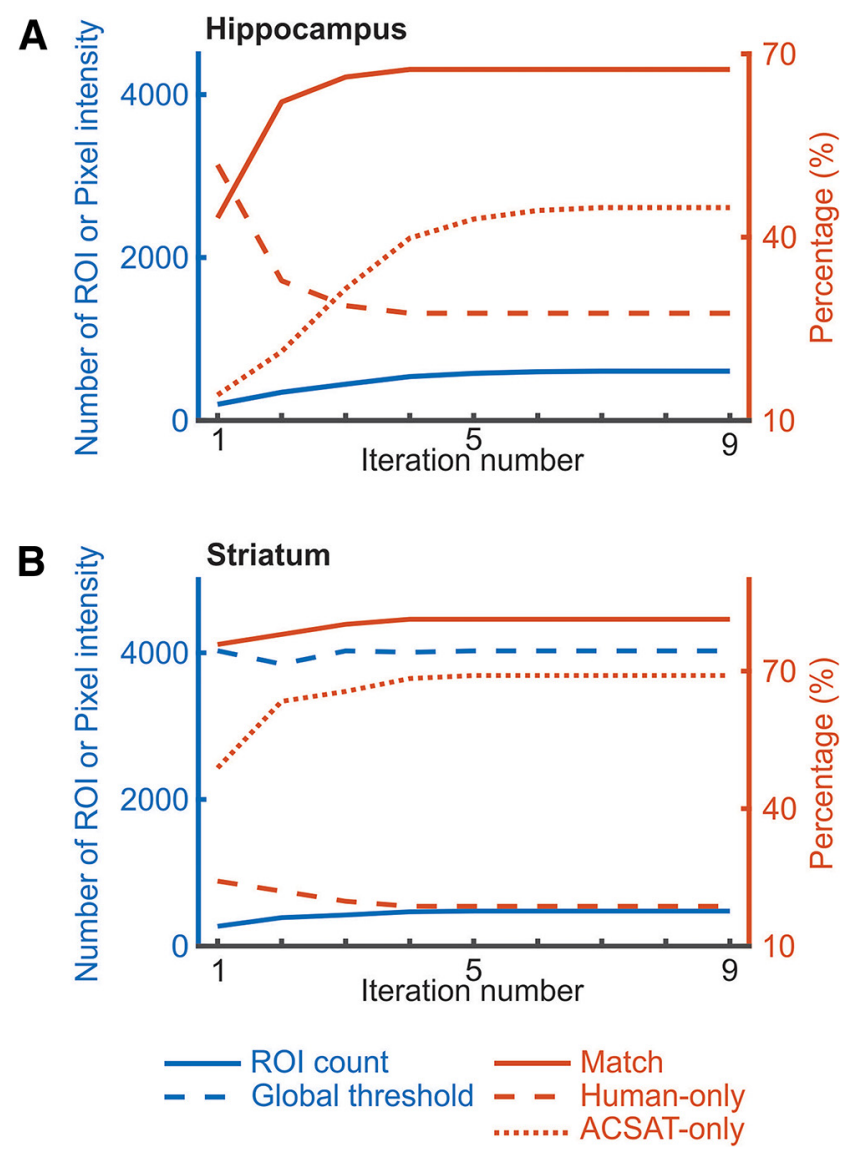

Figure 7. Performance of ACSAT over iterations. $\boldsymbol{A}, \boldsymbol{B}$, For both hippocampus dataset $A(\boldsymbol{A})$ and striatum dataset $(\boldsymbol{B})$, the cumulative number of identified ROls (solid line) increased steadily over iterations. The global threshold (dashed blue line) tended to decrease with each iteration, allowing ACSAT to capture ROIs with lower intensity. Both recall (solid red line) and false discovery rate $=1$ - precision (dotted red line) increased with iterations, while the false-negative rate (dashed red line) decreased. All results reported here are based on human-generated ROls before secondary manual inspection of false positives.

the whole intensity range at the fine scale, and, as a result, reduces the processing time.

After performing global thresholding to identify potential ROIs $\{R O / s\}_{n}^{\prime}$ (Fig. $1 A$ ), ACSAT further applies FIBAT locally to each identified $\mathrm{ROI}$ in $\{R O / s\}_{n}^{\prime}$ to refine the segmentation results (Fig. 9). When neurons are densely labeled with GCaMP6, using the global thresholding step alone may lead to one or more large clusters of adjacent neurons being segmented as a single ROI (Fig. 9A). For each such cluster, FIBAT (Fig. 1B) determines and applies a new threshold value to the local $\mathrm{ROI}$ area. With local thresholding, the example cluster is further segmented into five new ROls (Fig. 9B), which would not otherwise be separated by applying the global threshold. Because further local thresholding produces the same result (Fig. 9C), the local thresholding step of ACSAT concludes that these five ROls cannot be further separated, exits the recursive loop, and outputs these ROls.

To evaluate the efficacy of local thresholding, we examined the hippocampus dataset $A$ at each iteration be- 


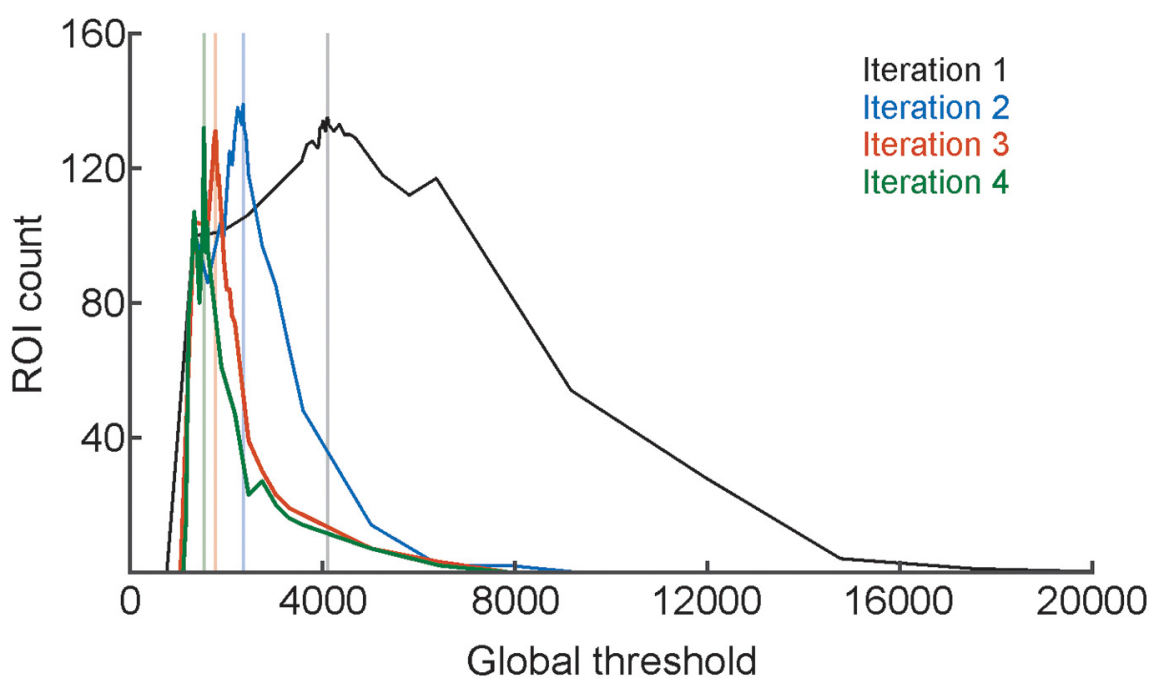

Figure 8. Convergence of the FIBAT optimal global threshold value for the hippocampus dataset A. FIBAT first sampled at a coarse scale across a wide intensity range, and then focused on a small potential intensity range with a fine scale to identify the optimal global threshold value that generated most ROIs. The vertical lines indicate the final optimal global threshold value determined by FIBAT for each iteration.

fore and after the local thresholding step (Fig. 10, left and right bars, respectively). Local thresholding refined the ROls detected by global thresholding and captured more true ROls at every iteration. It is also worth noting that, at later iterations, local thresholding was still able to identify true ROls that were missed by global thresholding alone (Fig. 10, iteration 4).

\section{ACSAT performance on two-photon dataset}

We applied ACSAT to the two-photon dataset Neurofinder 03.00 (Fig. 11C). Genetically Encoded Calcium
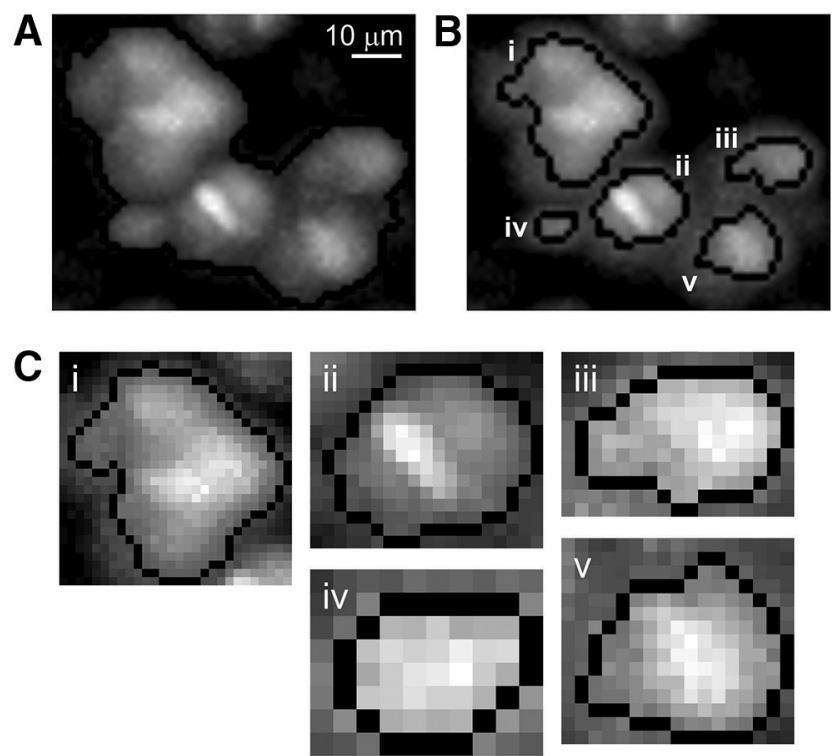

Figure 9. Improved ROI identification by local thresholding. $\boldsymbol{A}$, With global thresholding alone, a cluster of hippocampal neurons was identified as a single ROI. B, After application of local thresholding, ACSAT successfully separated five new ROls from the single ROI. C, Zoom-in of each ROI separated by local thresholding.
Indicators are generally not expressed in the nuclei (Tian et al., 2009), and because of the optical sectioning technique that two-photon imaging provides, in this dataset the nuclei appear dark. Additionally, this dataset had high speckle noise. Thus, the time-collapsed image generated by ACSAT using max minus mean pixel values shows bright nuclei. The truth file provided by Neurofinder contains 621 ROls, most of which are nuclei. Since the features of this dataset are the nuclei, which are smaller, we used the parameters $\delta=5 \%, A_{\min }=20 \mathrm{px} \approx 6.9 \mu \mathrm{m}^{2}$ and $A_{\max }=1000 \mathrm{px} \approx 34.6 \mu \mathrm{m}^{2}$ for the global adaptive thresholding step, and $A_{\min }=20 \mathrm{px} \approx 6.9 \mu \mathrm{m}^{2}$ and $A_{\max }=\infty$ for the local adaptive thresholding step.

ACSAT identified 571 ROls. Among these, 442 ROls were matched with the truth (true positive), and 179 ROls

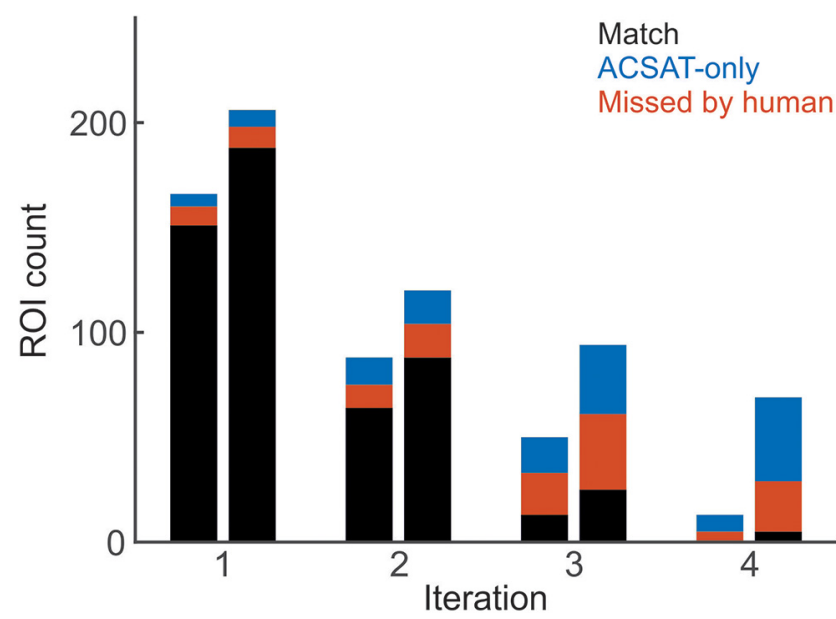

Figure 10. Local thresholding improves ACSAT performance for hippocampus dataset $A$. The ROls identified by ACSAT at each iteration before local thresholding (left) and after (right). Local thresholding separated overlapping ROls and thus helped identify more ROls, including those identified by human (black) or missed by human (red). 
A
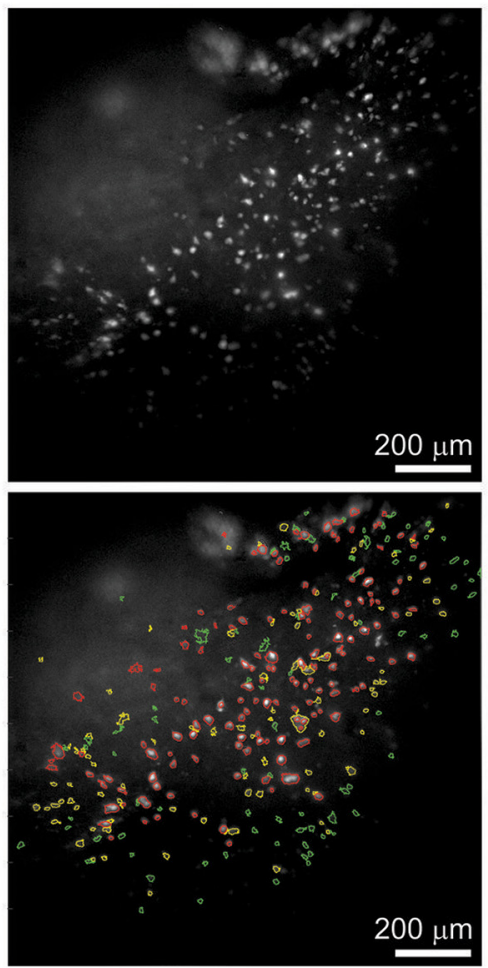

B
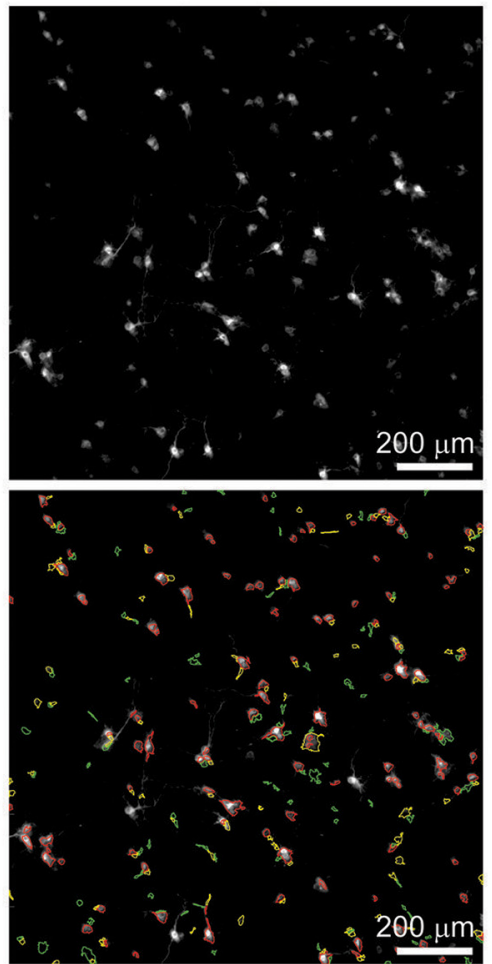

C

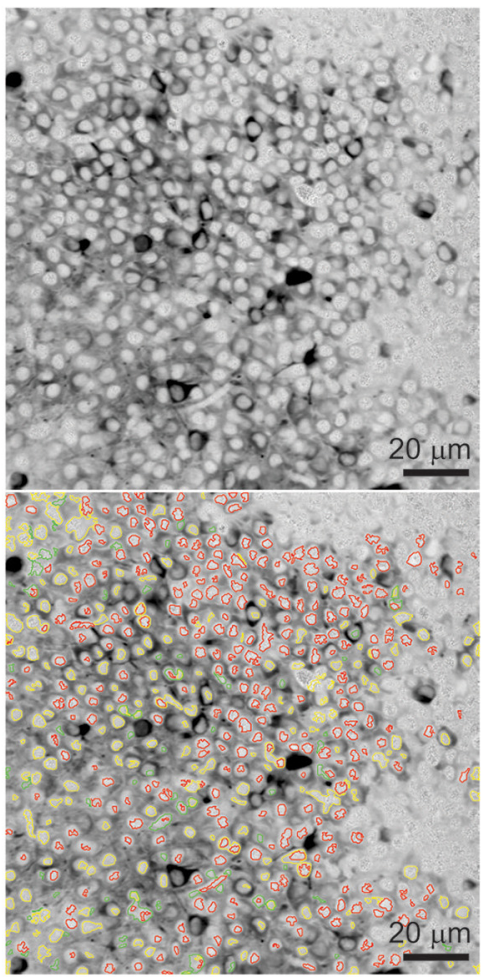

Figure 11. ACSAT results of various datasets. A, The time-collapsed image of hippocampus dataset B (top) with ACSAT ROIs overlaid (bottom). B, The time-collapsed image for the primary neuron culture dataset (top) with ACSAT ROls overlaid (bottom). $\boldsymbol{C}$, The time-collapsed image for the two-photon dataset (Neurofinder 03.00; top) with ACSAT ROls overlaid (bottom). For all three datasets, the majority of ROls were identified during the first two iterations. Red, yellow, green, and blue ROI outline corresponds to iteration $1,2,3$, and 4 , respectively.

were not in the truth (false positive). Additionally, 129 ROls in truth were not identified by ACSAT (false negative). This result gave us a recall rate of $71.2 \%$ (442 out of 621) and a precision rate of $77.4 \%$ (442 out of 571 ).

We further inspected the time-collapsed image $I_{0}$ and observed that the right side of the time-collapsed image $I_{0}$ had different patterns of texture than the left side. To use the new texture information for ROI detection by ACSAT, we extracted the right side of $I_{0}$ that is rich in texture information to generate $I_{0}^{\prime}$ as input to ACSAT. The $I_{0}^{\prime}$ was generated by change detection between the original image and its Gaussian-filtered counterpart. Thus, ACSAT identified an additional 157 ROls, of which 95 were true positives, and 62 were false positives. Combining these additional ROIs with the ROls identified by direct application of ACSAT results in a recall rate of $82.8 \%$ (514 out of 621 ) and a precision rate of $70.6 \%$ (514 out of 728 ).

\section{ACSAT performance on cell culture and hippocampus B dataset}

Finally, we used ACSAT to detect ROls in the dataset of the primary neuron culture expressing GCaMP6f (Fig. $11 B)$. Qualitatively, it appears ACSAT successfully identified the cell bodies of the majority of neurons in early iterations, and neurites in later iterations. We also used ACSAT to detect ROls in the hippocampus dataset B (Fig. $11 A$ ). For both datasets, we used the parameters $\delta=$ $10 \%, A_{\min }=50 \mathrm{px} \approx 86 \mu \mathrm{m}^{2}$ and $A_{\max }=300 \mathrm{px} \approx$
$516 \mu \mathrm{m}^{2}$ for the global adaptive thresholding step, and $A_{\min }=20 \mathrm{px} \approx 34 \mu \mathrm{m}^{2}$ and $A_{\max }=\infty$ for the local adaptive thresholding step because ROls tend to shrink in size after repeatedly applying FIBAT.

\section{Discussion}

In this study, we presented our automated cell segmentation by adaptive thresholding (ACSAT) method that adaptively selects threshold values based on image pixel intensity with two iterative steps at the global and local levels using a time-collapsed image. As such, the algorithm is capable of handling morphological variations in fluorescence intensity in neurons and is robust against luminance condition changes across datasets. When applied to two datasets collected from the hippocampus and the striatum in mice, ACSAT resulted in $\sim 80 \%$ recall rate of ROls containing individual neurons $(78.5 \%$ for the hippocampus $\mathrm{A}$ dataset and $82 \%$ for the striatum dataset), and $\sim 80 \%$ precision rate $(87 \%$ for the hippocampus dataset and $74.1 \%$ for the striatum dataset). ACSAT was also able to detect low-intensity ROls that were initially undetected by human referees. When applied to 500 simulated datasets, ACSAT achieved recall and precision rates higher than $80 \%$ when SNR was no less than $\sim 24$ dB. However, the performance of ACSAT falls when SNR reaches below $\sim 20 \mathrm{~dB}$.

The ACSAT algorithm is an intuitive thresholding method that uses global and local schemes to address variations in fluorescence intensity levels of GCaMP6 flu- 
orescence even within the same image field. Simply applying a lower global threshold value would result in few large ROls containing multiple neurons within one ROI. On the other hand, with a high global threshold value, only a small number of neurons with high intensity would be found. As such, applying a single high or low threshold value would generate inadequate results of either few or excessive ROls, which is a universal limitation of thresholding methods. Our algorithm efficiently addresses this challenge in two ways.

First, it cumulatively excludes previously segmented ROIs from the time-collapsed image $I_{n}$ after each iteration so that in the following iteration, ACSAT could detect new ROls that require distinct thresholds to separate but were missed with previous thresholds. Therefore, the global threshold value $\tau_{n}^{*}$ (Fig. 1) used by ACSAT usually decreases after each iteration, and ROls with high intensity were segmented before those with low intensity, as shown in Figs. 3 and 4. Because ACSAT is based on adaptive thresholding, it allows us to objectively and robustly segment ROls with low intensity relative to the background. These low-intensity areas often pose challenges to human experts when manually detecting ROIs, as our results showed that about half of the ROls initially labeled as false positive were actually true neurons (Fig. $10)$.

Second, ACSAT uses fluorescence intensity based adaptive thresholding (FIBAT) locally to separate overlapping ROIs. This approach directly addresses the issue of heterogeneity in recorded neural signals when the intensities of pixels surrounding an ROI can vary. However, because a higher thresholding value is usually required to separate adjoining neurons, the output sub-ROls after local FIBAT are often smaller than the corresponding true neurons. Thus, a simple dilation step was applied during the local FIBAT step. This correction is useful to prevent real ROIs from falling below the minimum area criterion $A_{\min }$ and thus being removed. Although the interleaving process of global FIBAT and local FIBAT has been effective in addressing overlapping neurons, a potential problem still arises if two neurons with similar intensities have significant overlap with each other in the time-collapsed image such that there is no trough between them. Then ACSAT may identify them as a single ROI. Conversely, if there is a neuron with multiple hotspots (Pnevmatikakis et al., 2016), then this may be identified as multiple neurons by ACSAT. Such a scenario, however, can be minimized by the minimum area criterion $A_{\min }$ and the maximum area criterion $A_{\max }$. Spatial overlap is profound for wide-field imaging, but not for two-photon imaging or in vitro cell culture imaging with single cell layer. With increasing improvement wide-field imaging, such as volumetric imaging (Shain et al., 2018; Xiao et al., 2018), such significant overlap may be better eliminated during data acquisition step.

ACSAT has three sets of free parameters that can be rationally chosen or otherwise are not sensitive: $\delta$, which describes the termination condition for ACSAT; $\alpha$, which describes a termination condition for FIBAT; and $A_{\min }$ and $A_{\max }$, which describe the allowed sizes of ROls.
The termination condition for ACSAT, described by $\delta$, can be explained by the tendencies of ACSAT. Specifically, running ACSAT for more iterations increases the number of ROls segmented, especially the number of low-intensity ROls, as the global threshold value $\tau_{n}^{*}$ gradually decreases (Fig. 7). While many of the added ROls are true ROls, the proportion of false-positive ROls added increases as iteration number increases (Fig. 7). This increasing proportion of outputted false positives in later iterations can be attributed to the higher probability of a spurious collection of adjacent background pixels meeting the criteria to be an ROI. Also, the added false positives can be related to the step which clears previously segmented ROls from the time-collapsed image at the start of each iteration of ACSAT. Due to the scattering of light in brain tissue, ROI removal may leave a few small fragments of bright pixels around removed areas, which could be identified as ROls during the next iteration. ACSAT tries to avoid this problem by dilating the cleared area, which makes sure the whole ROI is cleared rather than only the brighter center. Besides dilation, these misidentified ROls were also discarded either because of their small size or because they do not meet the solidity criteria; however, occasionally they may pass the size criteria and become the false-positive ROls. As a result, the majority of false positives tend to have small size (Fig. 6 , yellow).

To balance the effects of simultaneous increase in true ROls and false positive ROls, ACSAT stops when a decrease of global threshold value becomes relatively small between iterations, i.e.,

$$
\frac{\left|\tau_{n+1}^{*}-\tau_{n}^{*}\right|}{\tau_{1}^{*}}<\delta .
$$

At that stage, most true ROls have been detected and removed from the time-collapsed image. Thus, the global threshold values $\tau_{n}^{*}$ of any further iterations are similar, so most ROls detected at this stage are false positives. For the hippocampus dataset $A$, iteration $n=3$ is when the increase in false positives begins to outweigh the increase in true positives, and for the striatum dataset, nearly all true ROls segmented by ACSAT were outputted at iteration $n=1$ (Fig. 7). Qualitatively, the time-collapsed image $I_{0}$ for hippocampus has a higher density of neurons with a greater variety of pixel intensities than the $I_{0}$ for striatum, so it may take more iterations for ACSAT to perform at the same rate on the hippocampus dataset than on the striatum dataset. ACSAT's performance under the diverse conditions of these two datasets suggests that our choice of $\delta=10 \%$ provides a robust and rational termination condition for ACSAT that can be generalized to other datasets, namely the 500 simulated datasets and the cell culture dataset, as well. In fact, changing the termination condition from $\delta=10 \%$ to $\delta=5 \%$ only affected the segmentation results in $<17 \%$ of the 500 simulated datasets. For the two-photon dataset, our reported results are using the termination condition $\delta=5 \%$. In general, users can choose $\delta$ to be between $5 \%$ and $10 \%$ based on the needs of their application: if recall is more important, 
then users should choose a smaller $\delta$, and if precision is more important, then users should choose a larger $\delta$.

Additionally, the final segmentation results generated by ACSAT are not sensitive to the termination conditions for FIBAT described by $\alpha$ and $\varepsilon$. FIBAT is terminated if the threshold search range has minimal change over an iteration, which we determine in two ways. One way this condition would be satisfied is when all threshold values within the search range result in the same, optimal number of ROls. This is equivalent to setting the criterion $\alpha=100 \%$. For the practical purpose of reducing FIBAT run time, we allow termination if the change in the search range is $<1-\alpha=10 \%$. This condition is also easily met when FIBAT is used in the local thresholding step because, by definition, ROls that cannot be separated by FIBAT will return exactly one ROI no matter what threshold value is used. Additionally, we terminate FIBAT if the search range is smaller than $\varepsilon$, the smallest difference between any pair of adjacent pixels in $I$, which can be objectively and automatically determined from I. If FIBAT were to continue refining the threshold value, then the gained precision beyond that defined by $\varepsilon$ would be useless due to the discrete step in pixel intensity values in $I$.

The last set of parameters $A_{\min }$ and $A_{\max }$ should be chosen based on how large neurons are expected to be using information including neuron size, image resolution, magnification, imaging method, etc. In our wide-field datasets, the boundaries of neurons may not be as well defined as those collected with two-photon microscope, and the size will appear larger than the size of a neuron due to light scattering in wide-field conditions. This effect is consistent with our observation that the minimum size of the human-generated ROls was $38 \mathrm{px} \approx 64.6 \mu \mathrm{m}^{2}$ for the hippocampus $A$ dataset and $66 \mathrm{px} \approx 112.2 \mu \mathrm{m}^{2}$ for the striatum dataset. Thus, our minimum $\mathrm{ROI}$ criteria for the wide-field datasets may be larger than a typical neuron size.

The images $I_{0}$ used by ACSAT are time-collapsed, and therefore do not contain any temporal information. With the flexibility of ACSAT, the framework of ACSAT can be used as long as a single image can be generated to represent the ROls within the image sequence. For example, an input image $I_{0}^{\prime}$ can be generated where the value of each pixel represents the time of its maximum intensity. This image $I_{0}^{\prime}$ would allow ACSAT to separate adjoined ROIs that have similar intensity values in $I_{0}$ but reach their maximum intensity at different time points, which is described by $I_{0}^{\prime}$. Other ways to generate the single representative image include correlations with nearby pixels, intensity dynamics such as standard deviation or variance over time, texture of the time-collapsed image (for example, as used for the two-photon dataset), and a combination of various parameters. Overall, by taking advantage of adaptively determining the threshold value at both the global level and the local level, ACSAT can theoretically perform segmentation on any image containing ROls with nonhomogenous intensity as long as it has sufficient contrast between ROls and the background.

\section{References}

Allen WE, Kauvar IV, Chen MZ, Richman EB, Yang SJ, Chan K, Gradinaru V, Deverman BE, Luo L, Deisseroth K, (2017) Global representations of goal-directed behavior in distinct cell types of mouse neocortex. Neuron 94:891-907.e896. CrossRef

Andermann ML, Kerlin AM, Reid RC, (2010) Chronic cellular imaging of mouse visual cortex during operant behavior and passive viewing. Front Cell Neurosci 4:3. CrossRef Medline

Chen TW, Wardill TJ, Sun Y, Pulver SR, Renninger SL, Baohan A, Schreiter ER, Kerr RA, Orger MB, Jayaraman V, Looger LL, Svoboda K, Kim DS, (2013) Ultrasensitive fluorescent proteins for imaging neuronal activity. Nature 499:295-300. CrossRef Medline

Dombeck DA, Khabbaz AN, Collman F, Adelman TL, Tank DW, (2007) Imaging large-scale neural activity with cellular resolution in awake, mobile mice. Neuron 56:43-57. CrossRef Medline

Fantuzzo JA, Mirabella VR, Hamod AH, Hart RP, Zahn JD, Pang ZP, (2017) Intellicount: high-throughput quantification of fluorescent synaptic protein puncta by machine learning. eNeuro 4:021917.2017

Ghosh KK, Burns LD, Cocker ED, Nimmerjahn A, Ziv Y, Gamal AE, Schnitzer MJ (2011) Miniaturized integration of a fluorescence microscope. Nat Methods 8:871-878. CrossRef Medline

Hamel EJ, Grewe BF, Parker JG, Schnitzer MJ (2015) Cellular level brain imaging in behaving mammals: an engineering approach. Neuron 86:140-159. CrossRef Medline

Hofer SB, Ko H, Pichler B, Vogelstein J, Ros H, Zeng H, Lein E, Lesica NA, Mrsic-Flogel TD (2011) Differential connectivity and response dynamics of excitatory and inhibitory neurons in visual cortex. Nat Neurosci 14:1045-1052. CrossRef Medline

Huber D, Gutnisky DA, Peron S, O'Connor DH, Wiegert JS, Tian L, Oertner TG, Looger LL, Svoboda K (2012) Multiple dynamic representations in the motor cortex during sensorimotor learning. Nature 484:473-478. CrossRef Medline

Issa JB, Haeffele BD, Agarwal A, Bergles DE, Young ED, Yue DT (2014) Multiscale optical Ca2+ imaging of tonal organization in mouse auditory cortex. Neuron 83:944-959. CrossRef Medline

Kim TH, Zhang Y, Lecoq J, Jung JC, Li J, Zeng H, Niell CM, Schnitzer MJ (2016) Long-term optical access to an estimated one million neurons in the live mouse cortex. Cell Reports 17:3385-3394. CrossRef Medline

Lütcke H, Gerhard F, Zenke F, Gerstner W, Helmchen F (2013) Inference of neuronal network spike dynamics and topology from calcium imaging data. Front Neural Circuits 7:201. CrossRef Medline

Mellen NM, Tuong CM (2009) Semi-automated region of interest generation for the analysis of optically recorded neuronal activity. Neuroimage 47:1331-1340. CrossRef Medline

Mohammed Al, Gritton HJ, Tseng HA, Bucklin ME, Yao Z, Han X (2016) An integrative approach for analyzing hundreds of neurons in task performing mice using wide-field calcium imaging. Sci Rep 6:20986. CrossRef Medline

Moyer JR, Jr., Deyo RA, Disterhoft JF (1990) Hippocampectomy disrupts trace eye-blink conditioning in rabbits. Behav Neurosci 104:243-252. CrossRef

Mukamel EA, Nimmerjahn A, Schnitzer MJ (2009) Automated analysis of cellular signals from large-scale calcium imaging data. Neuron 63:747-760. CrossRef Medline

Ohki K, Chung S, Ch'ng YH, Kara P, Reid RC (2005) Functional imaging with cellular resolution reveals precise micro-architecture in visual cortex. Nature 433:597-603. CrossRef Medline

Otsu N (1979) Threshold selection method from gray-level histograms. leee T Syst Man Cyb 9:62-66. CrossRef

Pinto L, Dan Y (2015) Cell-type-specific activity in prefrontal cortex during goal-directed behavior. Neuron 87:437-450. CrossRef Medline

Pnevmatikakis EA, Soudry D, Gao Y, Machado TA, Merel J, Pfau D, Reardon T, Mu Y, Lacefield C, Yang W, Ahrens M, Bruno R, Jessell TM, Peterka DS, Yuste R, Paninski L (2016) Simultaneous denois- 
ing, deconvolution, and demixing of calcium imaging data. Neuron 89:285-299. CrossRef Medline

Poort J, Khan AG, Pachitariu M, Nemri A, Orsolic I, Krupic J, Bauza M, Sahani M, Keller GB, Mrsic-Flogel TD, Hofer SB (2015) Learning enhances sensory and multiple non-sensory representations in primary visual cortex. Neuron 86:1478-1490. CrossRef Medline

Sadeghian F, Seman Z, Ramli AR, Abdul Kahar BH, Saripan MI (2009) A framework for white blood cell segmentation in microscopic blood images using digital image processing. Biol Proced Online 11:196-206. CrossRef Medline

Sakamoto T, Takatsuki K, Kawahara S, Kirino Y, Niki H, Mishina M (2005) Role of hippocampal NMDA receptors in trace eyeblink conditioning. Brain Res 1039:130-136. CrossRef Medline

Sezgin M, Sankur B (2004) Survey over image thresholding techniques and quantitative performance evaluation. J Electron Imaging 13:146-168. CrossRef

Shain WJ, Vickers NA, Li J, Han X, Bifano T, Mertz J (2018) Axial localization with modulated-illumination extended-depth-of-field microscopy. Biomed Opt Express 9:1771-1782. CrossRef Medline

Shen S, Syal K, Tao N, Wang S (2015) An automated image analysis method for high-throughput classification of surface-bound bacterial cell motions. Rev Sci Instrum 86:126104. CrossRef Medline

Solomon PR, Vander Schaaf ER, Thompson RF, Weisz DJ (1986) Hippocampus and trace conditioning of the rabbit's classically conditioned nictitating membrane response. Behav Neurosci 100: 729-744. Medline

Sun XR, Badura A, Pacheco DA, Lynch LA, Schneider ER, Taylor MP, Hogue IB, Enquist LW, Murthy M, Wang SS (2013) Fast GCaMPs for improved tracking of neuronal activity. Nat Commun 4:2170. CrossRef Medline

Tian L, Hires SA, Mao T, Huber D, Chiappe ME, Chalasani SH, Petreanu L, Akerboom J, McKinney SA, Schreiter ER, Bargmann Cl, Jayaraman V, Svoboda K, Looger LL (2009) Imaging neural activity in worms, flies and mice with improved GCaMP calcium indicators. Nat Methods 6:875-881. CrossRef Medline

Tseng W, Guan R, Disterhoft JF, Weiss C (2004) Trace eyeblink conditioning is hippocampally dependent in mice. Hippocampus 14:58-65. CrossRef Medline

Wachowiak M, Economo MN, Díaz-Quesada M, Brunert D, Wesson DW, White JA, Rothermel M (2013) Optical dissection of odor information processing in vivo using GCaMPs expressed in specified cell types of the olfactory bulb. J Neurosci 33:5285-5300. CrossRef Medline

Wilt BA, Fitzgerald JE, Schnitzer MJ (2013) Photon shot noise limits on optical detection of neuronal spikes and estimation of spike timing. Biophys J 104:51-62. CrossRef Medline

Xiao S, Tseng HA, Gritton H, Han X, Mertz J (2018) Video-rate volumetric neuronal imaging using $3 \mathrm{D}$ targeted illumination. Sci Rep 8:7921. CrossRef Medline

Zhou P, Resendez SL, Rodriguez-Romaguera J, Jimenez JC, Neufeld SQ, Giovannucci A, Friedrich J, Pnevmatikakis EA, Stuber GD, Hen R, Kheirbek MA, Sabatini BL, Kass RE, Paninski L (2018) Efficient and accurate extraction of in vivo calcium signals from microendoscopic video data. eLife 7:e28728. CrossRef

Ziv Y, Burns LD, Cocker ED, Hamel EO, Ghosh KK, Kitch LJ, El Gamal A Schnitzer MJ (2013) Long-term dynamics of CA1 hippocampal place codes. Nat Neurosci 16:264-266. CrossRef Medline 